\title{
Rule based classifier for the analysis of gene-gene and gene-environment interactions in genetic association studies
}

\author{
Thorsten Lehr ${ }^{1,2^{*}}$, Jing Yuan ${ }^{2}$, Dirk Zeumer ${ }^{1}$, Supriya Jayadev ${ }^{2,3}$, Marylyn D Ritchie ${ }^{4}$
}

\author{
* Correspondence: thorsten. \\ lehr@boehringer-ingelheim.com \\ ${ }^{1}$ Boehringer Ingelheim Pharma \\ $\mathrm{GmbH} \&$ Co. KG, Department of \\ Drug Metabolism and \\ Pharmacokinetics, 88397 Biberach \\ an der Riss, Germany \\ Full list of author information is \\ available at the end of the article
}

\begin{abstract}
Background: Several methods have been presented for the analysis of complex interactions between genetic polymorphisms and/or environmental factors. Despite the available methods, there is still a need for alternative methods, because no single method will perform well in all scenarios. The aim of this work was to evaluate the performance of three selected rule based classifier algorithms, RIPPER, RIDOR and PART, for the analysis of genetic association studies.

Methods: Overall, 42 datasets were simulated with three different case-control models, a varying number of subjects $(300,600), \operatorname{SNPs}(500,1500,3000)$ and noise $(5 \%, 10 \%, 20 \%)$. The algorithms were applied to each of the datasets with a set of algorithm-specific settings. Results were further investigated with respect to a) the Model, b) the Rules, and c) the Attribute level. Data analysis was performed using WEKA, SAS and PERL.

Results: The RIPPER algorithm discovered the true case-control model at least once in $>33 \%$ of the datasets. The RIDOR and PART algorithm performed poorly for model detection. The RIPPER, RIDOR and PART algorithm discovered the true case-control rules in more than $83 \%, 83 \%$ and $44 \%$ of the datasets, respectively. All three algorithms were able to detect the attributes utilized in the respective case-control models in most datasets.

Conclusions: The current analyses substantiate the utility of rule based classifiers such as RIPPER, RIDOR and PART for the detection of gene-gene/gene-environment interactions in genetic association studies. These classifiers could provide a valuable new method, complementing existing approaches, in the analysis of genetic association studies. The methods provide an advantage in being able to handle both categorical and continuous variable types. Further, because the outputs of the analyses are easy to interpret, the rule based classifier approach could quickly generate testable hypotheses for additional evaluation. Since the algorithms are computationally inexpensive, they may serve as valuable tools for preselection of attributes to be used in more complex, computationally intensive approaches. Whether used in isolation or in conjunction with other tools, rule based classifiers are an important addition to the armamentarium of tools available for analyses of complex genetic association studies.
\end{abstract}




\section{Background}

Genetic association studies aim to identify the contribution of genetic polymorphisms to specific phenotypes such as disease status, drug responder status, and adverse drug reactions [1]. Association studies have been gaining interest as genotyping costs have significantly decreased and as more association-based success stories are reported [2,3].

In addition to the impact of a single genetic locus, more complex interactions between genetic polymorphisms and/or environmental factors, such as age, weight, and drug exposure can provide more accurate models for the prediction of complex phenotypes [4]. Several methods are currently available for the analysis of gene-gene and gene-environment interactions, e.g. random forests, focused interaction testing frameworks, stepwise logistic regression, explicit logistic regression, $\underline{\text { Multifactor }} \underline{\text { Dimension- }}$ ality Reduction (MDR) and Neural Networks (NN) [5,6].

One of the most frequently used algorithm, MDR, allows an exhaustive search for complex interactions [7]. MDR has a good sensitivity to detect gene-gene and geneenvironment interactions; however, the algorithm faces two challenges. First, large computational resources are required to perform the exhaustive searching, and the search for higher interaction models can be time consuming for large SNP panels (such as genome-wide association studies). Second, continuous variables have to be binned into categories to be considered for analysis, potentially leading to a loss of information. However, there are some approaches to alleviate this problem [8,9]. An alternative approach for the analysis of genetic association studies is the use of neural networks $(\mathrm{NN})$ and their modifications, e.g. genetic programming neural networks (GPNN) [10] and grammatical evolution neural networks (GENN) [11]. If well trained, neural network models yield good predictivity and allow for the incorporation of continuous variables. However, neural network models are often perceived to have a "black box character" where deconvoluting the contribution and interaction between genetic markers can be challenging. A recent comparison of methods showed that each method demonstrates strengths and weaknesses and there is still a need for alternative methods, because no single method will perform well in all scenarios [4].

Thus, rule based classifier algorithms which have proven performance with nongenetic datasets [12], could provide a valuable complementary method for the analysis of genetic association studies. Rule based classifiers generate classification models using a collection of "if ... then ..." rules [12]. The algorithms are computationally inexpensive, are capable of incorporating categorical and continuous variables and the developed models are usually easy to interpret.

The aim of the current study was to evaluate the performance of three selected rule based classifier algorithms, RIPPER [13], RIDOR [14] and PART [15], for the analysis of genetic association studies. Simulated datasets with varying statistical power and three different case-control models were generated in order to perform these evaluations.

\section{Methods}

\section{Algorithms}

Three different deterministic rule based classifier algorithms, for which the mathematical background is extensively described in the literature, were evaluated in the current study: (a) RIPPER (Repeated Incremental Pruning to Produce Error Reduction) [13] (b) RIDOR 
(RIpple-DOwn Rule) [14] and (c) PART [15]. The RIPPER algorithm is a direct method, i.e. RIPPER extracts the rules directly from the data. The algorithm progresses through four phases: i) growth, ii) pruning, iii) optimization, iv) selection. In the growth phase, one rule is generated by greedily adding attributes to the rule until the rule meets stopping criteria. In the following prune phase, each rule is incrementally pruned, allowing the pruning of any final sequence of the attributes, until a pruning metric is fulfilled. In the optimization stage each generated rule is further optimized by a) greedily adding attributes to the original rule and b) by independently growing a new rule undergoing a growth and pruning phase, as described above. Finally, in the selection phase, the best rules are kept and the other rules are deleted from the model. RIDOR is also a direct method, first generating a default rule (e.g. "all patients are controls") and then exceptions ("except if rs5 = $\mathrm{AB}$ and $\mathrm{rs} 10=\mathrm{AB}$ then patients are cases") to the default rule with the least error rate. The "best" exceptions for each exception are generated and iterated until pure. Thus, a tree-like expansion of exceptions is generated. The exceptions are a set of rules that predict classes other than the default. PART is an indirect method for rule generation. PART generates a pruned decision tree using the C4.5 statistical classifier [16] in each iteration. From the best tree, the leaves are translated into rules.

In the current study, the WEKA open-source software was used to implement the three rule-based classifier methods [17]. In WEKA, the RIPPER algorithm is implemented and named as JRIP (i.e. Java implementation of RIPPER).

\section{Datasets}

For the evaluation of the algorithms, three different case-control models (A, B \& C) (Table 1) were used for dataset simulation. Model A consisted of four case rules constructed by the interaction of two SNPs, rs5 and rs10. Model B consisted of two case rules constructed by the interaction of rs 5 and $\mathrm{rs} 10$ and by the interaction between SNP rs15 and the area under the curve (AUC) of a hypothetical compound X, as an environmental factor. For model $\mathrm{C}$ cases were assigned randomly without any relationship to a genotype or environmental factor, i.e. a "null model".

The case-control models A, B and C were used to simulate in total 42 various datasets: the number of subjects $(300,600)$, the number of SNPs $(500,1500,3000)$ and the noise of the case-control model with varying false positive (FP) and false negative (FN) rates $(5 \%, 10 \%, 20 \%)$ was varied for models $\mathrm{A}$ and $\mathrm{B}$ resulting in 18 different datasets for each case-control model (A and B) and 6 different datasets for model $\mathrm{C}$ (Table 2).

Table 1 Case-Control Models

\begin{tabular}{|c|c|c|}
\hline Model A & Model B & Model C \\
\hline Rule1: & Rule1: & Rule1: \\
\hline If $\mathrm{rs} 5=\mathrm{AA}$ and $\mathrm{rs} 10=\mathrm{AB}$ then Case & $\begin{array}{l}\text { If } \mathrm{rs} 5=\mathrm{BB} \text { and } \mathrm{rs} 10=\mathrm{AA} \\
\text { then Case }\end{array}$ & $\begin{array}{l}\text { If random number } \geq \text { "threshold" } \\
\text { then Case }\end{array}$ \\
\hline Rule2: & Rule2: & Rule 2: \\
\hline If $\mathrm{rs} 5=\mathrm{AB}$ and $\mathrm{rs} 10=\mathrm{AA}$ then Case & If rs $15=\mathrm{AA}$ and $\mathrm{AUC}>105$ then Case & Else Control \\
\hline Rule3: & Rule3: & \\
\hline If $r s 5=A B$ and $r s 10=B B$ then Case & Else Control & \\
\hline \multicolumn{3}{|l|}{ Rule4: } \\
\hline \multicolumn{3}{|l|}{ If $\mathrm{rs} 5=\mathrm{BB}$ and $\mathrm{rs} 10=\mathrm{AB}$ then Case } \\
\hline \multicolumn{3}{|l|}{ Rule 5: } \\
\hline Else Control & & \\
\hline
\end{tabular}


Table 2 Datasets investigated

\begin{tabular}{|c|c|c|c|c|c|c|}
\hline Dataset & Model & \# SNPs & \#Patients $\$$ & Ratio Control/Case & FP [\%] & FN [\%] \\
\hline 1 & $A$ & 500 & 300 & 2 & 5 & 5 \\
\hline 2 & A & 1500 & 300 & 2 & 5 & 5 \\
\hline 3 & A & 3000 & 300 & 2 & 5 & 5 \\
\hline 4 & A & 500 & 600 & 2 & 5 & 5 \\
\hline 5 & A & 1500 & 600 & 2 & 5 & 5 \\
\hline 6 & A & 3000 & 600 & 2 & 5 & 5 \\
\hline 7 & A & 500 & 300 & 2 & 10 & 10 \\
\hline 8 & A & 1500 & 300 & 2 & 10 & 10 \\
\hline 9 & A & 3000 & 300 & 2 & 10 & 10 \\
\hline 10 & A & 500 & 600 & 2 & 10 & 10 \\
\hline 11 & A & 1500 & 600 & 2 & 10 & 10 \\
\hline 12 & A & 3000 & 600 & 2 & 10 & 10 \\
\hline 13 & A & 500 & 300 & 2 & 20 & 20 \\
\hline 14 & A & 1500 & 300 & 2 & 20 & 20 \\
\hline 15 & A & 3000 & 300 & 2 & 20 & 20 \\
\hline 16 & A & 500 & 600 & 2 & 20 & 20 \\
\hline 17 & A & 1500 & 600 & 2 & 20 & 20 \\
\hline 18 & A & 3000 & 600 & 2 & 20 & 20 \\
\hline 19 & B & 500 & $150+150$ & 2 & 5 & 5 \\
\hline 20 & B & 1500 & $150+150$ & 2 & 5 & 5 \\
\hline 21 & B & 3000 & $150+150$ & 2 & 5 & 5 \\
\hline 22 & B & 500 & $300+300$ & 2 & 5 & 5 \\
\hline 23 & B & 1500 & $300+300$ & 2 & 5 & 5 \\
\hline 24 & B & 3000 & $300+300$ & 2 & 5 & 5 \\
\hline 25 & B & 500 & $150+150$ & 2 & 10 & 10 \\
\hline 26 & B & 1500 & $150+150$ & 2 & 10 & 10 \\
\hline 27 & B & 3000 & $150+150$ & 2 & 10 & 10 \\
\hline 28 & B & 500 & $300+300$ & 2 & 10 & 10 \\
\hline 29 & B & 1500 & $300+300$ & 2 & 10 & 10 \\
\hline 30 & B & 3000 & $300+300$ & 2 & 10 & 10 \\
\hline 31 & B & 500 & $150+150$ & 2 & 20 & 20 \\
\hline 32 & B & 1500 & $150+150$ & 2 & 20 & 20 \\
\hline 33 & B & 3000 & $150+150$ & 2 & 20 & 20 \\
\hline 34 & B & 500 & $300+300$ & 2 & 20 & 20 \\
\hline 35 & B & 1500 & $300+300$ & 2 & 20 & 20 \\
\hline 36 & B & 3000 & $300+300$ & 2 & 20 & 20 \\
\hline 37 & $C$ & 500 & 300 & 2 & n.a. & n.a. \\
\hline 38 & C & 1500 & 300 & 2 & n.a. & n.a. \\
\hline 39 & C & 3000 & 300 & 2 & n.a. & n.a. \\
\hline 40 & C & 500 & 600 & 2 & n.a. & n.a. \\
\hline 41 & C & 1500 & 600 & 2 & n.a. & n.a. \\
\hline 42 & C & 3000 & 600 & 2 & n.a. & n.a. \\
\hline
\end{tabular}

$\$$ For case rule $\mathrm{A}$ patients are equally distributed for the 4 case rules; for case rule $\mathrm{B}$ the first number indicates the number for rule 1 , the second number for rule 2 ; n.a: not applicable.

SNPs simulated in the datasets were named with a consecutive dbSNP [18] reference SNP identifier number (rs) from rs1 to rs1200. The reported rs numbers do not refer to their biological functionality as described in the dbSNP database [18]. For all other SNPs not used in the case-control models, no influence on the phenotype (i.e. case status) was assumed. The genotype frequencies were simulated randomly, uncorrelated and under assumption of Hardy-Weinberg Equilibrium (HWE) [19-21]. For the minor 
allele frequency (MAF) [22] a uniform distribution was selected ranging from $0-0.5$ with randomly varying minor alleles. The environmental factor AUC was simulated by a uniform distribution with medians at 110 and 95 resulting in a binomial distribution of the AUC. For the simulation of each of the 42 datasets different random seeds were used. Dataset generation was performed using SAS (SAS Institute Inc., Cary, NC, V 9.1.3). Simulated datasets are available upon request.

\section{Data Analysis}

Data analyses were performed using WEKA, version 3.7.0. For each algorithm, a varying set of algorithm specific options was applied resulting in 18, 30, and 9 different settings for RIPPER, PART and RIDOR, respectively (Table 3). The settings were chosen based on theoretical evaluations and based on previous experiences with the algorithms on similar datasets. Each of the settings (Table 3) was applied to each of the 42 simulated datasets (Table 2) using WEKA (command line mode). A customized Perl script

Table 3 Settings of Algorithm Options

\begin{tabular}{|c|c|c|c|}
\hline $\mathrm{Nr}$ & RIPPER & RIDOR & PART \\
\hline 1 & $-\mathrm{F} 3-\mathrm{N} 2.0-\mathrm{O} 10$ & $-\mathrm{F} 3-\mathrm{S} 1-\mathrm{N} 2.0-\mathrm{A}$ & $-R-B-M 2-N 3$ \\
\hline 2 & -F $3-\mathrm{N} 5.0-\mathrm{O} 10$ & -F 3 -S 1 -N 5.0 -A & $-R-B-M 5-N 3$ \\
\hline 3 & -F 3 -N $10.0-\mathrm{O} 10$ & $-\mathrm{F} 3-\mathrm{S} 1-\mathrm{N} 10.0-\mathrm{A}$ & $-R-B-M 10-N 3$ \\
\hline 4 & -F $10-\mathrm{N} 2.0-\mathrm{O} 10$ & $-\mathrm{F} 10-\mathrm{S} 1-\mathrm{N} 2.0-\mathrm{A}$ & $-R-B-M 2-N 10$ \\
\hline 5 & -F 10 -N $5.0-\mathrm{O} 10$ & -F $10-\mathrm{S} 1-\mathrm{N} 5.0-\mathrm{A}$ & $-R-B-M 5-N 10$ \\
\hline 6 & -F $10-\mathrm{N} 10.0-\mathrm{O} 10$ & -F 10 -S 1 -N $10.0-\mathrm{A}$ & $-\mathrm{R}-\mathrm{B}-\mathrm{M} 10-\mathrm{N} 10$ \\
\hline 7 & $-\mathrm{F} 100-\mathrm{N} 2.0-\mathrm{O} 10$ & $-\mathrm{F} 20-\mathrm{S} 1-\mathrm{N} 2.0-\mathrm{A}$ & $-R-B-M 2-N 100$ \\
\hline 8 & -F 100 -N $5.0-\mathrm{O} 10$ & -F 20 -S 1 -N $5.0-A$ & $-\mathrm{R}-\mathrm{B}-\mathrm{M} 5-\mathrm{N} 100$ \\
\hline 9 & -F $100-\mathrm{N} 10.0-\mathrm{O} 10$ & -F $20-\mathrm{S} 1-\mathrm{N} 10.0-\mathrm{A}$ & $-R-B-M 10-N 100$ \\
\hline 10 & -F 3 -N $2.0-O 100$ & & $-\mathrm{R}-\mathrm{M} 2-\mathrm{N} 3$ \\
\hline 11 & -F $3-\mathrm{N} 5.0-\mathrm{O} 100$ & & $-R-M 5-N 3$ \\
\hline 12 & -F 3 -N $10.0-0100$ & & $-R-M 10-N 3$ \\
\hline 13 & -F $10-\mathrm{N} 2.0-\mathrm{O} 100$ & & $-R-M 2-N 10$ \\
\hline 14 & -F $10-\mathrm{N} 5.0-\mathrm{O} 100$ & & -R $-\mathrm{M} 5-\mathrm{N} 10$ \\
\hline 15 & -F $10-\mathrm{N} 10.0-\mathrm{O} 100$ & & $-\mathrm{R}-\mathrm{M} 10-\mathrm{N} 10$ \\
\hline 16 & -F $100-\mathrm{N} 2.0-\mathrm{O} 100$ & & $-R-M 2-N 100$ \\
\hline 17 & -F 100 -N $5.0-\mathrm{O} 100$ & & -R -M 5 -N 100 \\
\hline 18 & -F $100-\mathrm{N} 10.0-\mathrm{O} 100$ & & $-R-M 10-N 100$ \\
\hline 19 & & & $-B-M 2-C 0.25$ \\
\hline 20 & & & -B -M 2 -C 0.1 \\
\hline 21 & & & $-B-M 5-C 0.25$ \\
\hline 22 & & & $-B-M 5-C 0.1$ \\
\hline 23 & & & -B -M 10 -C 0.25 \\
\hline 24 & & & $-B-M 10-C 0.1$ \\
\hline 25 & & & -M 2 -C 0.25 \\
\hline 26 & & & $-M 2-C 0.1$ \\
\hline 27 & & & -M 5 -C 0.25 \\
\hline 28 & & & $-M 5-C 0.1$ \\
\hline 29 & & & -M $10-C 0.25$ \\
\hline 30 & & & -M $10-C 0.1$ \\
\hline
\end{tabular}

RIPPER: F: number of folds for reduced error pruning; N: minimal weights of instances within a split; O: number of optimization runs.

RIDOR: F: number of folds for reduced error pruning; S: number of shuffles for randomization; A: Flag set to use the error rate of all the data to select the default class in each step. $\mathrm{N}$ : minimal weight of instances within a split.

PART: C: confidence threshold for pruning; $M$ : minimum number of instances per leaf; R: use reduced error pruning; $\mathrm{N}$ : number of folds for reduced error pruning; B: Use binary splits for nominal attributes. 
was used to extract the most important information (e.g. file name, model, statistics, etc.) from the WEKA result files and to summarize the information in a comma separated file. A grading system (A - D) was created (Table 4), to further compare the results at three different levels: a) the Model level, b) the Rules level, and c) the Attribute level.

On the Model level, each developed model was compared to the true model. At the Rules level, each rule was extracted individually from each developed model and compared to the true case-control rule. At both levels, the "best" grade was always assigned to each model, where A is better than B, B better than C, etc.

On the Attribute level, all SNPs and other attributes used as case-control predictors were extracted from the respective models. The appearance of each marker was counted for each dataset and algorithm. The most frequent marker was ranked as $1^{\text {st }}$, the second most frequent marker was ranked as $2^{\text {nd }}$, and so forth. Subsequently, the grading system was applied (Table 4). If the attribute ranking fulfilled a grading specification, the dataset and algorithm was assigned the "best" grade, where A is better than B, B better than C, etc. For the evaluation of the "null model" (model C), the same procedure as described above was applied. The true model was assumed to be either model A and/or model B. For the rule and attribute evaluation, the respective rules and attributes from models A and/or B were applied.

\section{Results}

In total, 2394 models ( $=42$ datasets $\times 57$ algorithm options) were generated and analyzed as described in the methods section. A summary statistic on the number of rules

Table 4 Grading System

\begin{tabular}{|c|c|c|c|}
\hline Grade & Model & Rules & Attribute \\
\hline$A$ & $\begin{array}{l}100 \% \text { accordance } \\
\text { Example: } \\
\text { If rs } 5=B B \text { and rs } 10=A A \text { then } \\
\text { Case; If rs } 15=A A \text { and } A \cup C>105 \\
\text { then Case; Else Control }\end{array}$ & $\begin{array}{l}100 \% \text { accordance } \\
\text { Example: } \\
\text { If } r 55=B B \text { and } r s 10=A A \\
\text { then Case }\end{array}$ & $\begin{array}{l}\text { All attributes were present (i.e. } \\
\text { detected) and ranked as most } \\
\text { frequent, i.e. top } 2 \text { for model A } \\
\text { and top } 4 \text { for model B. } \\
\text { Example: } \\
\text { rs } 5 \rightarrow \mathrm{Nr} .1 ; \text { rs } 10 \rightarrow \mathrm{Nr} \text {. 3; rs } 15 \rightarrow \\
\text { Nr.2; AUC } \rightarrow \mathrm{Nr} .4\end{array}$ \\
\hline$B$ & $\begin{array}{l}\text { One attribute was missing or an } \\
\text { additional attribute was identified } \\
\text { by the generated model } \\
\text { Example: } \\
\text { If } r s 5=B B \text { and rs } 10=A A \text { and rs } 234 \\
=B B \text { then Case; If rs } 15=A A \text { and } \\
\text { AUC }>105 \text { then Case; Else Control }\end{array}$ & $\begin{array}{l}\text { One attribute was missing or } \\
\text { an additional attribute was } \\
\text { identified by the generated } \\
\text { model } \\
\text { Example: } \\
\text { If } r 55=B B \text { and rs } 10=A A \text { and } \\
\text { rs } 234=B B \text { then Case }\end{array}$ & $\begin{array}{l}\text { All attributes were present but not } \\
\text { ranked as most frequent } \\
\text { Example: } \\
\text { rs5 } \rightarrow \text { Nr. 5; rs10 } \rightarrow \text { Nr. 3; rs15 } \rightarrow \\
\text { Nr.7; AUC } \rightarrow \text { Nr. } 4\end{array}$ \\
\hline$C$ & $\begin{array}{l}\text { Two attributes were different } \\
\text { between the generated and the } \\
\text { true model, } \\
\text { Example: } \\
\text { If rs } 5=B B \text { and rs } 10=A A \text { and } \\
\text { rs } 234=B B \text { then Case; If rs } 15=A A \\
\text { and } A U C>105 \text { and rs } 56=A A \text { then } \\
\text { Case; Else Control }\end{array}$ & $\begin{array}{l}\text { Two attributes were different } \\
\text { between the generated and } \\
\text { the true model } \\
\text { Example: } \\
\text { If rs } 5=B B \text { and rs } 234=B B \\
\text { then Case }\end{array}$ & $\begin{array}{l}\text { One attribute was not present, } \\
\text { remaining attributes were present } \\
\text { and rank was not considered } \\
\text { Example: } \\
\text { rs } 5 \rightarrow \text { not detected; rs } 10, \text { rs } 15 \text { and } \\
\text { AUC } \rightarrow \text { detected }\end{array}$ \\
\hline $\mathrm{D}$ & $\begin{array}{l}\text { Three attributes were different } \\
\text { between the generated and the } \\
\text { true model } \\
\text { Example: } \\
\text { If rs } 5=B B \text { and rs } 234=B B \text { and } r 56= \\
A A \text { then Case; If rs } 15=A A \text { and } A U C \\
>105 \text { then Case; Else Control }\end{array}$ & $\begin{array}{l}\text { Three attributes were } \\
\text { different between the } \\
\text { generated and the true } \\
\text { model } \\
\text { Example: } \\
\text { If } r s 5=B B \text { and rs } 56=A A \text { and } \\
\text { rs } 234=B B \text { then Case }\end{array}$ & $\begin{array}{l}\text { Two attributes were not present, } \\
\text { remaining attributes were present } \\
\text { and ranked as most frequent } \\
\text { Example: } \\
\text { rs5 and AUC } \rightarrow \text { not detected; rs } 10 \\
\text { and rs } 15 \rightarrow \text { detected }\end{array}$ \\
\hline
\end{tabular}

Comparison of the generated versus true model, rules and attributes. (Model B was used for the example.) 
per model and the number of attributes per model is presented in Table 5. A qualitative summary of the results is shown in Table 6 . Detailed quantitative results at the Model, Rule and Attribute level are presented in Tables 7, 8, \& 9.

\section{Models Level Analyses}

The models generated had in median 4 to 47 unique attributes per model (Table 5). The RIPPER algorithm utilized the fewest number of attributes (median: 4 to 15) whereas RIDOR and PART had a significantly higher number of attributes per model (median: 16 to 47). The number of attributes was approximately two times higher for the "null model" (model C) compared to models A and B. The number of rules per model was comparable between all three algorithms (median: 3 to 9.5).

The RIPPER algorithm performed well and was able to discover the true model (Grade A) at least once, 33\% of the time with case-control model A and $56 \%$ of the time with case-control model B (Table 6). The RIDOR and PART algorithm performed poorly in model detection. Case-control model A was not discovered in any dataset by the two algorithms. Case-control model B was identified at least once in 11\% and 33\% of the datasets by RIDOR and PART, respectively.

When minor deviations from the true model were considered (Grades B - D), RIPPER was again able to detect significantly more models compared to the RIDOR and PART algorithms. Overall, case-control model B was better discovered by all three algorithms, compared to case-control model A. None of the methods detected for model $\mathrm{C}$ a false positive finding.

Table 7 provides a detailed summary of model level algorithm performance. In general, algorithms performed worse if the power of the dataset decreased, i.e. less subjects and/or more SNPs and/or higher FP/FN rates.

\section{Rules Level Analyses}

At the rules level, the RIPPER algorithm performed well and was able to discover the true case rules (Grade A) at least once, $83 \%$ of the time with case-control model A and 100\% of the time with case-control model B (Table 6). The RIDOR and PART algorithms also

Table 5 Statistics

\begin{tabular}{|c|c|c|c|c|c|c|c|c|c|c|}
\hline & & \multicolumn{3}{|c|}{ RIPPER } & \multicolumn{3}{|c|}{ RIDOR } & \multicolumn{3}{|c|}{ PART } \\
\hline & & $\begin{array}{c}\text { Model } \\
\text { A }\end{array}$ & $\begin{array}{c}\text { Model } \\
\text { B }\end{array}$ & $\begin{array}{l}\text { Model } \\
\text { C }\end{array}$ & $\begin{array}{c}\text { Model } \\
\text { A }\end{array}$ & $\begin{array}{c}\text { Model } \\
\text { B }\end{array}$ & $\begin{array}{l}\text { Model } \\
\text { C }\end{array}$ & $\begin{array}{c}\text { Model } \\
\text { A }\end{array}$ & $\begin{array}{l}\text { Model } \\
\text { B }\end{array}$ & $\begin{array}{l}\text { Model } \\
\text { C }\end{array}$ \\
\hline \# & Min & 1 & 3 & 1 & 2 & 3 & 1 & 1 & 1 & 2 \\
\hline \multirow[t]{4}{*}{$\begin{array}{l}\text { Attributes* per } \\
\text { model }\end{array}$} & $\begin{array}{l}\text { 5th } \\
\text { Percentile }\end{array}$ & 2 & 3 & 1 & 4 & 5 & 4 & 2 & 4 & 7 \\
\hline & Median & 7 & 4 & 15 & 16 & 17 & 28 & 24 & 24 & 47 \\
\hline & $\begin{array}{l}\text { 95th } \\
\text { Percentile }\end{array}$ & 28 & 13 & 42 & 46 & 50 & 70 & 102 & 109 & 144 \\
\hline & Max & 43 & 21 & 63 & 65 & 62 & 73 & 245 & 240 & 259 \\
\hline \multirow{5}{*}{$\begin{array}{l}\text { \# rules }{ }^{5} \text { per } \\
\text { model }\end{array}$} & Min & 2 & 3 & 2 & 3 & 3 & 1 & 1 & 1 & 1 \\
\hline & $\begin{array}{l}\text { 5th } \\
\text { Percentile }\end{array}$ & 3 & 3 & 2 & 4 & 4 & 2 & 2 & 2 & 1 \\
\hline & Median & 6 & 3 & 6 & 8 & 8 & 9.5 & 7 & 6 & 9 \\
\hline & $\begin{array}{l}\text { 95th } \\
\text { Percentile }\end{array}$ & 11 & 7 & 14 & 16 & 17 & 19 & 25.5 & 24.5 & 37.5 \\
\hline & Max & 16 & 9 & 18 & 20 & 20 & 20 & 45 & 43 & 46 \\
\hline
\end{tabular}

* unique SNP or unique environmental variable; ${ }^{5}$ Case and control rules combined. 
Table 6 Qualitative Results - Summary

\begin{tabular}{|c|c|c|c|c|c|c|c|c|c|c|}
\hline \multirow[b]{2}{*}{ Evaluation Level } & \multirow[b]{2}{*}{ Grade } & \multicolumn{3}{|c|}{ RIPPER } & \multicolumn{3}{|c|}{ RIDOR } & \multicolumn{3}{|c|}{ PART } \\
\hline & & $\begin{array}{c}\text { Model } \\
\text { A }\end{array}$ & Model B & Model C & $\begin{array}{c}\text { Model } \\
\text { A }\end{array}$ & Model B & Model C & $\begin{array}{c}\text { Model } \\
\text { A }\end{array}$ & Model B & Model C \\
\hline \multirow[t]{4}{*}{ Models } & $A$ & $33 \%(6)$ & $56 \%(10)$ & $0 \%(0)$ & $0 \%(0)$ & $11 \%(2)$ & $0 \%(0)$ & $0 \%(0)$ & $33 \%(6)$ & $0 \%(0)$ \\
\hline & B & $33 \%(6)$ & $50 \%(9)$ & $0 \%(0)$ & $0 \%(0)$ & $6 \%(1)$ & $0 \%(0)$ & $0 \%(0)$ & $33 \%(6)$ & $0 \%(0)$ \\
\hline & C & $22 \%(4)$ & $56 \%(10)$ & $0 \%(0)$ & $0 \%(0)$ & $6 \%(1)$ & $0 \%(0)$ & $0 \%(0)$ & $22 \%(4)$ & $0 \%(0)$ \\
\hline & D & $6 \%(1)$ & $22 \%(4)$ & $0 \%(0)$ & $0 \%(0)$ & $17 \%(3)$ & $0 \%(0)$ & $6 \%(1)$ & $11 \%(2)$ & $0 \%(0)$ \\
\hline \multirow[t]{4}{*}{ Rules } & A & $83 \%(15)$ & $100 \%(18)$ & $0 \%(0)$ & $83 \%(15)$ & $94 \%(17)$ & $0 \%(0)$ & $44 \%(8)$ & $78 \%(14)$ & $0 \%(0)$ \\
\hline & B & $89 \%(16)$ & $72 \%(13)$ & $0 \%(0)$ & $94 \%(17)$ & $100 \%(18)$ & $0 \%(0)$ & $50 \%(9)$ & $100 \%(18)$ & $0 \%(0)$ \\
\hline & C & $67 \%(12)$ & $67 \%(12)$ & $0 \%(0)$ & $56 \%(10)$ & $100 \%(18)$ & $0 \%(0)$ & $78 \%(14)$ & $89 \%(16)$ & $0 \%(0)$ \\
\hline & D & $50 \%(9)$ & $56 \%(10)$ & $0 \%(0)$ & $67 \%(12)$ & $100 \%(18)$ & $0 \%(0)$ & $56 \%(10)$ & $94 \%(17)$ & $0 \%(0)$ \\
\hline \multirow[t]{4}{*}{ Attributes } & A & $83 \%(15)$ & $56 \%(10)$ & $0 \%(0)$ & $50 \%(9)$ & $72 \%(13)$ & $0 \%(0)$ & $11 \%(2)$ & $0 \%(0)$ & $0 \%(0)$ \\
\hline & B & 17\% (3) & $28 \%(5)$ & $0 \%(0)$ & $50 \%(9)$ & $17 \%(3)$ & $0 \%(0)$ & $61 \%(11)$ & $78 \%(14)$ & $0 \%(0)$ \\
\hline & C & $0 \%(0)$ & 17\% (3) & $0 \%(0)$ & $0 \%(0)$ & $11 \%(2)$ & $0 \%(0)$ & $6 \%(1)$ & $22 \%(4)$ & $0 \%(0)$ \\
\hline & D & $0 \%(0)$ & $0 \%(0)$ & $0 \%(0)$ & $0 \%(0)$ & $0 \%(0)$ & $0 \%(0)$ & $22 \%(4)$ & $0 \%(0)$ & $0 \%(0)$ \\
\hline
\end{tabular}

Summary of the results, separated by algorithm, case-control model, and grading. Number represents the percent frequency of datasets where a respective grade was achieved at least once (absolute number is in brackets). 
Table 7 Quantitative Results - Models Level

\begin{tabular}{|c|c|c|c|c|c|c|c|c|c|c|c|c|c|c|c|c|}
\hline \multirow[b]{2}{*}{ Model } & \multirow[b]{2}{*}{ Dataset } & \multirow[b]{2}{*}{ SNPs } & \multirow[b]{2}{*}{ Patients } & \multirow[b]{2}{*}{ Error [\%] } & \multicolumn{4}{|c|}{ RIPPER } & \multicolumn{4}{|c|}{ RIDOR } & \multicolumn{4}{|c|}{ PART } \\
\hline & & & & & A & B & $\mathrm{C}$ & D & A & B & $C$ & D & A & B & $C$ & D \\
\hline A & 1 & 500 & 300 & 5 & 0 & 1 & 7 & 0 & 0 & 0 & 0 & 0 & 0 & 0 & 0 & 0 \\
\hline A & 2 & 1500 & 300 & 5 & 0 & 0 & 0 & 0 & 0 & 0 & 0 & 0 & 0 & 0 & 0 & 2 \\
\hline A & 3 & 3000 & 300 & 5 & 0 & 0 & 0 & 0 & 0 & 0 & 0 & 0 & 0 & 0 & 0 & 0 \\
\hline A & 4 & 500 & 600 & 5 & 3 & 1 & 0 & 0 & 0 & 0 & 0 & 0 & 0 & 0 & 0 & 0 \\
\hline A & 5 & 1500 & 600 & 5 & 7 & 5 & 0 & 0 & 0 & 0 & 0 & 0 & 0 & 0 & 0 & 0 \\
\hline A & 6 & 3000 & 600 & 5 & 1 & 2 & 2 & 0 & 0 & 0 & 0 & 0 & 0 & 0 & 0 & 0 \\
\hline A & 7 & 500 & 300 & 10 & 0 & 0 & 0 & 0 & 0 & 0 & 0 & 0 & 0 & 0 & 0 & 0 \\
\hline A & 8 & 1500 & 300 & 10 & 0 & 1 & 0 & 0 & 0 & 0 & 0 & 0 & 0 & 0 & 0 & 0 \\
\hline A & 9 & 3000 & 300 & 10 & 0 & 0 & 0 & 0 & 0 & 0 & 0 & 0 & 0 & 0 & 0 & 0 \\
\hline A & 10 & 500 & 600 & 10 & 8 & 0 & 1 & 0 & 0 & 0 & 0 & 0 & 0 & 0 & 0 & 0 \\
\hline A & 11 & 1500 & 600 & 10 & 0 & 0 & 0 & 7 & 0 & 0 & 0 & 0 & 0 & 0 & 0 & 0 \\
\hline A & 12 & 3000 & 600 & 10 & 1 & 0 & 1 & 0 & 0 & 0 & 0 & 0 & 0 & 0 & 0 & 0 \\
\hline A & 13 & 500 & 300 & 20 & 0 & 0 & 0 & 0 & 0 & 0 & 0 & 0 & 0 & 0 & 0 & 0 \\
\hline A & 14 & 1500 & 300 & 20 & 0 & 0 & 0 & 0 & 0 & 0 & 0 & 0 & 0 & 0 & 0 & 0 \\
\hline A & 15 & 3000 & 300 & 20 & 0 & 0 & 0 & 0 & 0 & 0 & 0 & 0 & 0 & 0 & 0 & 0 \\
\hline A & 16 & 500 & 600 & 20 & 6 & 3 & 0 & 0 & 0 & 0 & 0 & 0 & 0 & 0 & 0 & 0 \\
\hline A & 17 & 1500 & 600 & 20 & 0 & 0 & 0 & 0 & 0 & 0 & 0 & 0 & 0 & 0 & 0 & 0 \\
\hline A & 18 & 3000 & 600 & 20 & 0 & 0 & 0 & 0 & 0 & 0 & 0 & 0 & 0 & 0 & 0 & 0 \\
\hline B & 19 & 500 & $150+150$ & 5 & 11 & 7 & 0 & 0 & 2 & 0 & 1 & 0 & 1 & 1 & 0 & 0 \\
\hline B & 20 & 1500 & $150+150$ & 5 & 15 & 0 & 3 & 0 & 0 & 1 & 0 & 0 & 2 & 0 & 0 & 0 \\
\hline B & 21 & 3000 & $150+150$ & 5 & 4 & 4 & 2 & 0 & 0 & 0 & 0 & 3 & 0 & 0 & 1 & 1 \\
\hline B & 22 & 500 & $300+300$ & 5 & 14 & 0 & 4 & 0 & 0 & 0 & 0 & 0 & 5 & 0 & 0 & 0 \\
\hline B & 23 & 1500 & $300+300$ & 5 & 18 & 0 & 0 & 0 & 0 & 0 & 0 & 0 & 0 & 2 & 0 & 0 \\
\hline B & 24 & 3000 & $300+300$ & 5 & 17 & 1 & 0 & 0 & 0 & 0 & 0 & 0 & 1 & 1 & 0 & 0 \\
\hline B & 25 & 500 & $150+150$ & 10 & 7 & 10 & 0 & 0 & 0 & 0 & 0 & 0 & 1 & 0 & 2 & 0 \\
\hline B & 26 & 1500 & $150+150$ & 10 & 0 & 2 & 2 & 0 & 0 & 0 & 0 & 1 & 0 & 1 & 2 & 1 \\
\hline B & 27 & 3000 & $150+150$ & 10 & 0 & 3 & 2 & 1 & 0 & 0 & 0 & 0 & 0 & 0 & 0 & 0 \\
\hline B & 28 & 500 & $300+300$ & 10 & 18 & 0 & 0 & 0 & 1 & 0 & 0 & 0 & 1 & 1 & 0 & 0 \\
\hline B & 29 & 1500 & $300+300$ & 10 & 17 & 1 & 0 & 0 & 0 & 0 & 0 & 0 & 0 & 1 & 1 & 0 \\
\hline B & 30 & 3000 & $300+300$ & 10 & 13 & 1 & 0 & 0 & 0 & 0 & 0 & 0 & 0 & 0 & 0 & 0 \\
\hline B & 31 & 500 & $150+150$ & 20 & 0 & 0 & 7 & 1 & 0 & 0 & 0 & 1 & 0 & 0 & 0 & 0 \\
\hline B & 32 & 1500 & $150+150$ & 20 & 0 & 0 & 1 & 0 & 0 & 0 & 0 & 0 & 0 & 0 & 0 & 0 \\
\hline B & 33 & 3000 & $150+150$ & 20 & 0 & 0 & 4 & 4 & 0 & 0 & 0 & 0 & 0 & 0 & 0 & 0 \\
\hline B & 34 & 500 & $300+300$ & 20 & 0 & 4 & 1 & 0 & 0 & 0 & 0 & 0 & 0 & 0 & 0 & 0 \\
\hline B & 35 & 1500 & $300+300$ & 20 & 0 & 0 & 1 & 0 & 0 & 0 & 0 & 0 & 0 & 0 & 0 & 0 \\
\hline B & 36 & 3000 & $300+300$ & 20 & 0 & 0 & 0 & 7 & 0 & 0 & 0 & 0 & 0 & 0 & 0 & 0 \\
\hline C & 37 & 500 & 300 & n.a. & 0 & 0 & 0 & 0 & 0 & 0 & 0 & 0 & 0 & 0 & 0 & 0 \\
\hline C & 38 & 1500 & 300 & n.a. & 0 & 0 & 0 & 0 & 0 & 0 & 0 & 0 & 0 & 0 & 0 & 0 \\
\hline C & 39 & 3000 & 300 & n.a. & 0 & 0 & 0 & 0 & 0 & 0 & 0 & 0 & 0 & 0 & 0 & 0 \\
\hline C & 40 & 500 & 600 & n.a. & 0 & 0 & 0 & 0 & 0 & 0 & 0 & 0 & 0 & 0 & 0 & 0 \\
\hline C & 41 & 1500 & 600 & n.a. & 0 & 0 & 0 & 0 & 0 & 0 & 0 & 0 & 0 & 0 & 0 & 0 \\
\hline C & 42 & 3000 & 600 & n.a. & 0 & 0 & 0 & 0 & 0 & 0 & 0 & 0 & 0 & 0 & 0 & 0 \\
\hline
\end{tabular}

Summary of the results at the model level, separated by algorithm, case-control model, dataset and grading. Number expresses the absolute frequency of the respective grading assignment.

performed well in rule detection. For case-control model A, the true rules were discovered at least once $83 \%$ and $44 \%$ of the time with the RIDOR and PART algorithms, respectively. With case-control model B the RIDOR and PART algorithms were able to discover the true rule at least once in $94 \%$ and $78 \%$ of the datasets, respectively. 
Table 8 Quantitative Results - Rules Level

\begin{tabular}{|c|c|c|c|c|c|c|c|c|c|c|c|c|c|c|c|c|}
\hline \multirow[b]{2}{*}{ Model } & \multirow[b]{2}{*}{ Dataset } & \multirow[b]{2}{*}{ SNPs } & \multirow[b]{2}{*}{ Patients } & \multirow[b]{2}{*}{ Error [\%] } & \multicolumn{4}{|c|}{ RIPPER } & \multicolumn{4}{|c|}{ RIDOR } & \multicolumn{4}{|c|}{ PART } \\
\hline & & & & & A & B & $C$ & D & A & B & $C$ & D & A & B & $C$ & D \\
\hline A & 1 & 500 & 300 & 5 & 44 & 14 & 3 & 0 & 4 & 6 & 2 & 3 & 12 & 13 & 2 & 2 \\
\hline A & 2 & 1500 & 300 & 5 & 0 & 36 & 0 & 0 & 4 & 16 & 0 & 3 & 3 & 4 & 1 & 2 \\
\hline$A$ & 3 & 3000 & 300 & 5 & 0 & 1 & 7 & 0 & 0 & 1 & 0 & 0 & 0 & 0 & 0 & 0 \\
\hline A & 4 & 500 & 600 & 5 & 46 & 14 & 0 & 0 & 30 & 20 & 0 & 0 & 25 & 28 & 27 & 10 \\
\hline A & 5 & 1500 & 600 & 5 & 72 & 0 & 0 & 0 & 12 & 29 & 4 & 1 & 2 & 4 & 3 & 2 \\
\hline A & 6 & 3000 & 600 & 5 & 28 & 32 & 0 & 0 & 8 & 27 & 0 & 0 & 12 & 6 & 2 & 1 \\
\hline A & 7 & 500 & 300 & 10 & 23 & 27 & 4 & 7 & 2 & 5 & 0 & 1 & 2 & 3 & 2 & 0 \\
\hline A & 8 & 1500 & 300 & 10 & 19 & 13 & 0 & 3 & 2 & 12 & 0 & 2 & 2 & 0 & 2 & 0 \\
\hline A & 9 & 3000 & 300 & 10 & 1 & 11 & 12 & 11 & 0 & 1 & 3 & 3 & 0 & 0 & 0 & 0 \\
\hline$A$ & 10 & 500 & 600 & 10 & 46 & 16 & 0 & 0 & 11 & 23 & 7 & 0 & 25 & 20 & 11 & 2 \\
\hline A & 11 & 1500 & 600 & 10 & 35 & 10 & 15 & 6 & 6 & 19 & 11 & 2 & 0 & 11 & 16 & 9 \\
\hline A & 12 & 3000 & 600 & 10 & 48 & 5 & 20 & 0 & 7 & 7 & 0 & 0 & 0 & 0 & 3 & 1 \\
\hline A & 13 & 500 & 300 & 20 & 11 & 21 & 8 & 3 & 1 & 4 & 3 & 6 & 0 & 0 & 4 & 2 \\
\hline A & 14 & 1500 & 300 & 20 & 5 & 1 & 6 & 9 & 0 & 1 & 3 & 0 & 0 & 0 & 0 & 0 \\
\hline A & 15 & 3000 & 300 & 20 & 3 & 0 & 1 & 0 & 1 & 0 & 0 & 7 & 0 & 0 & 0 & 0 \\
\hline A & 16 & 500 & 600 & 20 & 48 & 17 & 7 & 1 & 8 & 6 & 17 & 4 & 0 & 2 & 1 & 0 \\
\hline A & 17 & 1500 & 600 & 20 & 25 & 18 & 26 & 2 & 8 & 4 & 15 & 4 & 0 & 0 & 2 & 4 \\
\hline A & 18 & 3000 & 600 & 20 & 0 & 19 & 1 & 3 & 1 & 6 & 2 & 4 & 0 & 0 & 1 & 0 \\
\hline$B$ & 19 & 500 & $150+150$ & 5 & 29 & 4 & 3 & 0 & 8 & 13 & 5 & 5 & 31 & 17 & 9 & 2 \\
\hline B & 20 & 1500 & $150+150$ & 5 & 30 & 6 & 0 & 0 & 8 & 8 & 7 & 5 & 12 & 13 & 19 & 3 \\
\hline B & 21 & 3000 & $150+150$ & 5 & 27 & 5 & 13 & 8 & 7 & 5 & 11 & 7 & 4 & 21 & 8 & 8 \\
\hline B & 22 & 500 & $300+300$ & 5 & 36 & 0 & 0 & 0 & 8 & 35 & 9 & 3 & 41 & 41 & 15 & 10 \\
\hline B & 23 & 1500 & $300+300$ & 5 & 36 & 0 & 0 & 0 & 9 & 21 & 12 & 5 & 20 & 27 & 11 & 4 \\
\hline B & 24 & 3000 & $300+300$ & 5 & 35 & 1 & 0 & 0 & 9 & 19 & 10 & 10 & 13 & 22 & 25 & 8 \\
\hline B & 25 & 500 & $150+150$ & 10 & 25 & 11 & 1 & 1 & 4 & 4 & 17 & 20 & 12 & 20 & 14 & 1 \\
\hline B & 26 & 1500 & $150+150$ & 10 & 16 & 5 & 19 & 9 & 1 & 9 & 14 & 7 & 17 & 13 & 3 & 3 \\
\hline B & 27 & 3000 & $150+150$ & 10 & 18 & 4 & 11 & 14 & 0 & 14 & 3 & 9 & 0 & 5 & 1 & 1 \\
\hline B & 28 & 500 & $300+300$ & 10 & 36 & 0 & 0 & 0 & 17 & 8 & 9 & 8 & 28 & 18 & 14 & 10 \\
\hline B & 29 & 1500 & $300+300$ & 10 & 35 & 1 & 0 & 0 & 7 & 14 & 16 & 6 & 7 & 27 & 7 & 4 \\
\hline B & 30 & 3000 & $300+300$ & 10 & 31 & 7 & 2 & 0 & 7 & 9 & 12 & 9 & 2 & 17 & 14 & 7 \\
\hline B & 31 & 500 & $150+150$ & 20 & 18 & 0 & 13 & 10 & 4 & 3 & 9 & 5 & 0 & 1 & 3 & 2 \\
\hline B & 32 & 1500 & $150+150$ & 20 & 10 & 8 & 21 & 11 & 2 & 8 & 5 & 6 & 0 & 1 & 0 & 0 \\
\hline B & 33 & 3000 & $150+150$ & 20 & 9 & 7 & 18 & 2 & 1 & 2 & 7 & 9 & 0 & 1 & 0 & 1 \\
\hline B & 34 & 500 & $300+300$ & 20 & 18 & 17 & 3 & 13 & 4 & 5 & 18 & 13 & 2 & 1 & 6 & 1 \\
\hline B & 35 & 1500 & $300+300$ & 20 & 18 & 0 & 9 & 31 & 4 & 3 & 12 & 13 & 2 & 4 & 7 & 2 \\
\hline B & 36 & 3000 & $300+300$ & 20 & 16 & 2 & 23 & 20 & 5 & 3 & 5 & 8 & 1 & 3 & 1 & 1 \\
\hline C & 37 & 500 & 300 & n.a. & 0 & 0 & 0 & 0 & 0 & 0 & 0 & 0 & 0 & 0 & 0 & 0 \\
\hline C & 38 & 1500 & 300 & n.a. & 0 & 0 & 0 & 0 & 0 & 0 & 0 & 0 & 0 & 0 & 0 & 0 \\
\hline C & 39 & 3000 & 300 & n.a. & 0 & 0 & 0 & 0 & 0 & 0 & 0 & 0 & 0 & 0 & 0 & 0 \\
\hline C & 40 & 500 & 600 & n.a. & 0 & 0 & 0 & 0 & 0 & 0 & 0 & 0 & 0 & 0 & 0 & 0 \\
\hline C & 41 & 1500 & 600 & n.a. & 0 & 0 & 0 & 0 & 0 & 0 & 0 & 0 & 0 & 0 & 0 & 0 \\
\hline C & 42 & 3000 & 600 & n.a. & 0 & 0 & 0 & 0 & 0 & 0 & 0 & 0 & 0 & 0 & 0 & 0 \\
\hline
\end{tabular}

Summary of the results at the rules level, separated by algorithm, case-control model, dataset and grading. Number expresses the absolute frequency of the respective grading assignment.

When deviations (Grades B - D) from the true case rules were allowed, all algorithms performed with high discovery rates. Overall the case rules of case-control model B were slightly better discovered by all three algorithms, compared to the case rules of case-control model A. None of the methods detected for the rules of model $\mathrm{C}$ a false positive finding. 
Table 9 Quantitative Results - Attributes Level

\begin{tabular}{|c|c|c|c|c|c|c|c|c|c|c|c|c|c|c|c|c|}
\hline \multirow[b]{2}{*}{ Model } & \multirow[b]{2}{*}{ Dataset } & \multirow[b]{2}{*}{ SNPs } & \multirow[b]{2}{*}{ Patients } & \multirow[b]{2}{*}{ Error [\%] } & \multicolumn{4}{|c|}{ RIPPER } & \multicolumn{4}{|c|}{ RIDOR } & \multicolumn{4}{|c|}{ PART } \\
\hline & & & & & A & B & $C$ & D & A & B & $C$ & D & A & B & $C$ & D \\
\hline A & 1 & 500 & 300 & 5 & 1 & 0 & 0 & 0 & 0 & 1 & 0 & 0 & 0 & 1 & 0 & 0 \\
\hline A & 2 & 1500 & 300 & 5 & 1 & 0 & 0 & 0 & 1 & 0 & 0 & 0 & 0 & 1 & 0 & 0 \\
\hline A & 3 & 3000 & 300 & 5 & 0 & 1 & 0 & 0 & 0 & 1 & 0 & 0 & 0 & 0 & 0 & 0 \\
\hline A & 4 & 500 & 600 & 5 & 1 & 0 & 0 & 0 & 1 & 0 & 0 & 0 & 1 & 0 & 0 & 1 \\
\hline A & 5 & 1500 & 600 & 5 & 1 & 0 & 0 & 0 & 1 & 0 & 0 & 0 & 0 & 1 & 0 & 0 \\
\hline A & 6 & 3000 & 600 & 5 & 1 & 0 & 0 & 0 & 1 & 0 & 0 & 0 & 0 & 1 & 0 & 0 \\
\hline A & 7 & 500 & 300 & 10 & 1 & 0 & 0 & 0 & 0 & 1 & 0 & 0 & 0 & 1 & 0 & 0 \\
\hline A & 8 & 1500 & 300 & 10 & 1 & 0 & 0 & 0 & 1 & 0 & 0 & 0 & 0 & 1 & 0 & 0 \\
\hline A & 9 & 3000 & 300 & 10 & 1 & 0 & 0 & 0 & 0 & 1 & 0 & 0 & 0 & 0 & 0 & 1 \\
\hline A & 10 & 500 & 600 & 10 & 1 & 0 & 0 & 0 & 1 & 0 & 0 & 0 & 1 & 0 & 0 & 0 \\
\hline A & 11 & 1500 & 600 & 10 & 1 & 0 & 0 & 0 & 1 & 0 & 0 & 0 & 0 & 1 & 0 & 0 \\
\hline A & 12 & 3000 & 600 & 10 & 1 & 0 & 0 & 0 & 0 & 1 & 0 & 0 & 0 & 1 & 0 & 0 \\
\hline A & 13 & 500 & 300 & 20 & 1 & 0 & 0 & 0 & 0 & 1 & 0 & 0 & 0 & 1 & 0 & 0 \\
\hline A & 14 & 1500 & 300 & 20 & 0 & 1 & 0 & 0 & 0 & 1 & 0 & 0 & 0 & 0 & 0 & 1 \\
\hline A & 15 & 3000 & 300 & 20 & 0 & 1 & 0 & 0 & 0 & 1 & 0 & 0 & 0 & 0 & 0 & 1 \\
\hline A & 16 & 500 & 600 & 20 & 1 & 0 & 0 & 0 & 1 & 0 & 0 & 0 & 0 & 1 & 0 & 0 \\
\hline A & 17 & 1500 & 600 & 20 & 1 & 0 & 0 & 0 & 1 & 0 & 0 & 0 & 0 & 1 & 0 & 0 \\
\hline A & 18 & 3000 & 600 & 20 & 1 & 0 & 0 & 0 & 0 & 1 & 0 & 0 & 0 & 0 & 1 & 0 \\
\hline B & 19 & 500 & $150+150$ & 5 & 1 & 0 & 0 & 0 & 1 & 0 & 0 & 0 & 0 & 1 & 0 & 0 \\
\hline B & 20 & 1500 & $150+150$ & 5 & 1 & 0 & 0 & 0 & 1 & 0 & 0 & 0 & 0 & 1 & 0 & 0 \\
\hline B & 21 & 3000 & $150+150$ & 5 & 1 & 0 & 0 & 0 & 1 & 0 & 0 & 0 & 0 & 1 & 0 & 0 \\
\hline B & 22 & 500 & $300+300$ & 5 & 1 & 0 & 0 & 0 & 1 & 0 & 0 & 0 & 0 & 1 & 0 & 0 \\
\hline B & 23 & 1500 & $300+300$ & 5 & 1 & 0 & 0 & 0 & 1 & 0 & 0 & 0 & 0 & 1 & 0 & 0 \\
\hline B & 24 & 3000 & $300+300$ & 5 & 1 & 0 & 0 & 0 & 0 & 1 & 0 & 0 & 0 & 1 & 0 & 0 \\
\hline B & 25 & 500 & $150+150$ & 10 & 1 & 0 & 0 & 0 & 0 & 0 & 1 & 0 & 0 & 1 & 0 & 0 \\
\hline B & 26 & 1500 & $150+150$ & 10 & 0 & 1 & 0 & 0 & 1 & 0 & 0 & 0 & 0 & 1 & 0 & 0 \\
\hline B & 27 & 3000 & $150+150$ & 10 & 0 & 0 & 1 & 0 & 0 & 0 & 1 & 0 & 0 & 1 & 0 & 0 \\
\hline B & 28 & 500 & $300+300$ & 10 & 1 & 0 & 0 & 0 & 1 & 0 & 0 & 0 & 0 & 1 & 0 & 0 \\
\hline B & 29 & 1500 & $300+300$ & 10 & 1 & 0 & 0 & 0 & 1 & 0 & 0 & 0 & 0 & 1 & 0 & 0 \\
\hline B & 30 & 3000 & $300+300$ & 10 & 1 & 0 & 0 & 0 & 1 & 0 & 0 & 0 & 0 & 1 & 0 & 0 \\
\hline B & 31 & 500 & $150+150$ & 20 & 0 & 1 & 0 & 0 & 1 & 0 & 0 & 0 & 0 & 0 & 1 & 0 \\
\hline B & 32 & 1500 & $150+150$ & 20 & 0 & 0 & 1 & 0 & 0 & 1 & 0 & 0 & 0 & 0 & 1 & 0 \\
\hline B & 33 & 3000 & $150+150$ & 20 & 0 & 0 & 1 & 0 & 0 & 1 & 0 & 0 & 0 & 0 & 1 & 0 \\
\hline B & 34 & 500 & $300+300$ & 20 & 0 & 1 & 0 & 0 & 1 & 0 & 0 & 0 & 0 & 1 & 0 & 0 \\
\hline B & 35 & 1500 & $300+300$ & 20 & 0 & 1 & 0 & 0 & 1 & 0 & 0 & 0 & 0 & 1 & 0 & 0 \\
\hline B & 36 & 3000 & $300+300$ & 20 & 0 & 1 & 0 & 0 & 1 & 0 & 0 & 0 & 0 & 0 & 1 & 0 \\
\hline C & 37 & 500 & 300 & n.a. & 0 & 0 & 0 & 0 & 0 & 0 & 0 & 0 & 0 & 0 & 0 & 0 \\
\hline C & 38 & 1500 & 300 & n.a. & 0 & 0 & 0 & 0 & 0 & 0 & 0 & 0 & 0 & 0 & 0 & 0 \\
\hline C & 39 & 3000 & 300 & n.a. & 0 & 0 & 0 & 0 & 0 & 0 & 0 & 0 & 0 & 0 & 0 & 0 \\
\hline C & 40 & 500 & 600 & n.a. & 0 & 0 & 0 & 0 & 0 & 0 & 0 & 0 & 0 & 0 & 0 & 0 \\
\hline C & 41 & 1500 & 600 & n.a. & 0 & 0 & 0 & 0 & 0 & 0 & 0 & 0 & 0 & 0 & 0 & 0 \\
\hline C & 42 & 3000 & 600 & n.a. & 0 & 0 & 0 & 0 & 0 & 0 & 0 & 0 & 0 & 0 & 0 & 0 \\
\hline
\end{tabular}

Summary of the results at the attribute level, separated by algorithm, case-control model, dataset and grading. A "1" reflects an affiliation to the respective grading, whereas a "0" reflects no affiliation. 
Table 8 provides a detailed summary of rule level algorithm performance. All algorithms performed worse in rule detection if the power of the dataset decreased, i.e. less subjects and/or more SNPs and/or higher FP/FN rates.

\section{Attributes Level Analyses}

At the attributes level, the RIPPER algorithm performed well. RIPPER yielded an "A" grade (all attributes present and ranked as the top 2 (model A) or the top 4 (model B) attributes) in $83 \%$ of the time with case-control model $\mathrm{A}$ and $56 \%$ of the time with case-control model B (Table 6). The RIDOR algorithm demonstrated satisfactory performance at the attribute level with $50 \%$ and $72 \%$ of the datasets yielding an "A" grade in case-control models A and B, respectively. The PART algorithm did not perform as well. With case-control model A, the grade "A" designation occurred only $11 \%$ of the time and with case-control model $B$, the "A" grade did not get assigned to any of the outputs. For model C, all grading (A-D) were zero across all methods.

Table 9 provides a detailed summary of attribute level algorithm performance. All algorithms performed worse in attribute detection, if the power of the dataset decreased, i.e. less subjects and/or more SNPs and/or higher FP/FN rates.

\section{Discussion}

At the model level, the RIPPER algorithm outperformed the other two evaluated algorithms. RIPPER discovered the true model (grade "A") or a slight variation of the true model (grade "B") $>40 \%$ of the time with case-control model A and $>70 \%$ of the time with case-control model B. The RIDOR and PART algorithms were not able to detect the true case-control model A. Even under less stringent conditions of evaluation (Grade B), allowing minor deviations from the true model, the two algorithms were not able to discover the true case-control model. For case-control model B the RIDOR and PART algorithms performed better, however, RIPPER still outperformed the two. In general, the RIDOR and PART algorithms tended to build overly complex prediction models with a median of 2 to 3 times more predictors compared to the RIPPER algorithm. This complexity was probably caused by the nature of the algorithm. The PART algorithm derives rules from decision trees and decision trees tend to build overly complex models [12]. With the RIDOR algorithm, the "exception from the exception" principle is utilized for data investigation, this may not be appropriate to analyze complex gene-gene or gene-environment interactions.

All three algorithms performed well in detecting case-control rules. As with model level analyses, the RIPPER algorithm was superior to the RIDOR and PART algorithms in rule level analyses. The RIPPER algorithm discovered rules at a much higher frequency, even when corrected for the number of options tested (Table 3). If the frequency of each rule is counted and the rules ordered according to their frequencies (analogous to the attribute level analyses), the RIPPER algorithm identified more topranked rules than the other two algorithms.

At the attribute level, the RIPPER algorithm performed slightly better than RIDOR. The RIPPER algorithm discovered and top-ranked the true attributes $83 \%$ and $56 \%$ of the time for case-control models A and B, respectively. In contrast, the RIDOR algorithm discovered and top-ranked the true attributes $50 \%$ and $72 \%$ of the time for case-control models $\mathrm{A}$ and $\mathrm{B}$, respectively. The PART algorithm performed the worst of the three algorithms but still identified the true attributes $72 \%$ and $78 \%$ of the time for case-control models A 
and $\mathrm{B}$, respectively. The PART algorithm did not rank the true attributes in the top 2 (model A) or 4 (model B) as consistently as the RIPPER and RIDOR algorithms.

In addition to the two case-control models $\mathrm{A}$ and $\mathrm{B}$ datasets without relationships between genotypes and phenotypes (model C, "null model") were also tested. The null model results were not useful in further discriminating between the three rule based classifiers. For all datasets and with all methods, no false positive number of models, attributes or rules were identified. Nevertheless, the models derived from the "null model" datasets showed that the median number of attributes per model was twice as high compared to the real case-control models A and B. The number of attributes per model might therefore be a mechanism to discriminate true models from false positive models.

The RIPPER algorithm appears to be superior to the PART and RIDOR algorithm at all levels: models, rules and attributes. The tested set of algorithm options (Table 3) provides an adequate toolset for comparison; however, this work could be expanded and further optimized using simulated and non-simulated data.

Based on the analysis presented, questions may arise on how to best translate the rule based classifier into practice and how to apply the classifiers to non-simulated data. In data analysis practice, one would apply a selected classifier, preferably RIPPER, to one dataset with a battery of options, e.g. as provided in Table 3. Thus, for RIPPER this would result in 18 different models, one for each setting. For each of the 18 models standard statistics, e.g. numbers of rules, sensitivity, specificity, accuracy, etc., are provided. The next steps would be mainly triggered by the purpose of the analysis, depending on whether the analyst aims to use the classifiers as model builders or as filters. If the classifier is to be used for model building, the revealed models should be further investigated, e.g. by thorough review considering statistics such as sensitivity, specificity and complexity of the models. If classifiers are to be used as filters for attributes, similar procedures as described in the methods section could be applied. Additional research is required regarding practical considerations and evaluation methods, such as cross validation or external prediction.

In the presented analyses, the size of the datasets tested was limited to 3000 SNPs and 600 patients. Technically, rule based classifiers are neither limited by number of SNPs nor by number of patients. Thus, the methods should be scalable to whole genome levels. However, the size of the dataset analyzed in WEKA may be limited by the available computational memory and has to be taken into account.

\section{Conclusions}

The current analyses substantiate the utility of rule based classifiers such as RIPPER, RIDOR and PART for the detection of gene-gene and gene-environment interactions in genetic association studies. These methods could provide a valuable new method, complementing existing approaches, in the analysis of genetic association studies. The methods provide an advantage in being able to handle both categorical and continuous variable types, and since the outputs of the analyses are easy to interpret the rule based classifier approach could quickly generate testable hypotheses for further evaluation. In addition, since the algorithms are computationally inexpensive to run, they may serve as valuable tools for preselection of attributes to be used in more complex, computationally intensive approaches such as MDR. Whether used in isolation or in conjunction with other tools, rule based classifiers are an important addition to the armamentarium of tools available for analyses of complex genetic association studies. 
As a next step, the most promising algorithm RIPPER should be benchmarked against other popular analysis methods, such as MDR or Random Forests.

\title{
Acknowledgements
}

The authors would like to thank Dr. Sheng Guo and Dr. Paul Tanswell for the critical review of the manuscript and the valuable comments.

\begin{abstract}
Author details
${ }^{1}$ Boehringer Ingelheim Pharma GmbH \& Co. KG, Department of Drug Metabolism and Pharmacokinetics, 88397 Biberach an der Riss, Germany. ${ }^{2}$ Boehringer Ingelheim Pharma Inc., Department of Toxicology and Safety Assessment, 900 Ridgebury Road, Ridgefield, CT 06877, USA. ${ }^{3}$ Boehringer Ingelheim Pharma Inc., Department of Research Operations, 900 Ridgebury Road, Ridgefield, CT 06877, USA. ${ }^{4}$ Vanderbilt University Medical Center, Department of Molecular Physiology \& Biophysics, Center for Human Genetics Research, 519 Light Hall, Nashville, TN 37232, USA.
\end{abstract}

\section{Authors' contributions}

$T L$ designed the analysis, established the algorithms, performed the analysis and drafted the manuscript. JY contributed to the design of the simulated data and the scientific framing of queries to be performed. DZ developed the Perl summary scripts and contributed to the design of the analysis. SJ contributed to the establishment of the algorithms and the manuscript. MDR participated in the design and coordination of the analysis and helped to draft the manuscript. All authors read and approved the final manuscript.

\section{Competing interests}

$T L, D Z, J Y, S J$ and MDR have no competing interests.

Received: 6 November 2009 Accepted: 1 March 2011 Published: 1 March 2011

\section{References}

1. Lewis CM: Genetic association studies: design, analysis and interpretation. Briefings in bioinformatics 2002, 3:146-153.

2. Hirschhorn JN, Lohmueller K, Byrne E, Hirschhorn K: A comprehensive review of genetic association studies. Genetics in Medicine 2002, 4:45-61.

3. Cordell HJ, Clayton DG: Genetic association studies. Lancet 2005, 366:1121-1131.

4. Thornton-Wells TA, Moore JH, Haines JL: Genetics, statistics and human disease: Analytical retooling for complexity. Trends in Genetics 2004, 20:640-647.

5. Moore JH: Analysis of gene-gene interactions. Current Protocols in Human Genetics 2008.

6. Motsinger-Reif AA, Reif DM, Fanelli TJ, Ritchie MD: A comparison of analytical methods for genetic association studies. Genet Epidemio/ 2008, 32:767-778.

7. Hahn LW, Ritchie MD, Moore JH: Multifactor dimensionality reduction software for detecting gene-gene and geneenvironment interactions. Bioinformatics 2003, 19:376-382.

8. Calle ML, Urrea V, Vellalta G, Malats N, Steen KV: Improving strategies for detecting genetic patterns of disease susceptibility in association studies. Statistics in medicine 2008, 27:6532-6546.

9. Lou XY, Chen GB, Yan L, Ma JZ, Zhu J, Elston RC, Li MD: A generalized combinatorial approach for detecting geneby-gene and gene-by-environment interactions with application to nicotine dependence. Amer J Hum Genet 2007, 80:1125-1137.

10. Ritchie MD, White BC, Parker JS, Hahn LW, Moore JH: Optimization of neural network architecture using genetic programming improves detection and modelling of gene-gene interactions in studies of human diseases. BMC Bioinformatics 2003, 4.

11. Motsinger AA, Reif DM, Dudek SM, Ritchie MD: Understanding the evolutionary process of grammatical evolution neural networks for feature selection in genetic epidemiology.263-270.

12. Tan PN, Steinbach M, Kumar V: Introduction to Data Mining Addison-Wesley; 2006.

13. Cohen W: Fast Effective Rule Induction. Twelfth International Conference on Machine Learning Morgan Kaufmann; 1995, 115-123.

14. Gaines B, Compton P: Induction of Ripple-Down Rules Applied to Modeling Large Databases. J Intell Inf Syst 1995, 5:211-228.

15. Frank E, Witten I: Generating Accurate Rule Sets Without Global Optimization. In Fifteenth International Conference on Machine Learning Edited by: Shavlik J, Morgan Kaufmann 1998, 144-151.

16. Quinlan R: C4.5: Programs for Machine Learning San Mateo, CA: Morgan Kaufmann Publishers; 1993.

17. Witten I, Frank E: Data Mining: Practical machine learning tools and techniques San Francisco: Morgan Kaufmann; 2005.

18. Sherry ST, Ward MH, Kholodov M, Baker J, Phan L, Smigielski EM, Sirotkin K: DbSNP: The NCBI database of genetic variation. Nucl Acids Res 2001, 29:308-311.

19. Schaap T: The applicability of the Hardy-Weinberg principle in the study of populations. Ann Hum Genet 1980, 44:211-215.

20. Louis EJ, Dempster ER: An exact test for Hardy-Weinberg and multiple alleles. Biometrics 1987, 43:805-811.

21. Bourgain C, Abney M, Schneider D, Ober C, McPeek MS: Testing for Hardy-Weinberg equilibrium in samples with related individuals. Genetics 2004, 168:2349-2361.

22. Goddard KAB, Hopkins PJ, Hall JM, Witte JS: Linkage disequilibrium and allele-frequency distributions for 114 singlenucleotide polymorphisms five populations. Amer J Hum Genet 2000, 66:216-234.

doi:10.1186/1756-0381-4-4

Cite this article as: Lehr et al:: Rule based classifier for the analysis of gene-gene and gene-environment

interactions in genetic association studies. BioData Mining 2011 4:4. 